Diffusion Induced Chaos in a Closed Loop Thermosyphon

Author(s): Aníbal Rodríguez-Bernal and Erik S. van Vleck

Reviewed work(s):

Source: SIAM Journal on Applied Mathematics, Vol. 58, No. 4 (Aug., 1998), pp. 1072-1093

Published by: Society for Industrial and Applied Mathematics

Stable URL: http://www.jstor.org/stable/118320

Accessed: 08/10/2012 07:13

Your use of the JSTOR archive indicates your acceptance of the Terms \& Conditions of Use, available at http://www.jstor.org/page/info/about/policies/terms.jsp

JSTOR is a not-for-profit service that helps scholars, researchers, and students discover, use, and build upon a wide range of content in a trusted digital archive. We use information technology and tools to increase productivity and facilitate new forms of scholarship. For more information about JSTOR, please contact support@jstor.org. 


\title{
DIFFUSION INDUCED CHAOS IN A CLOSED LOOP THERMOSYPHON*
}

\author{
ANÍBAL RODRÍGUEZ-BERNAL ${ }^{\dagger}$ AND ERIK S. VAN VLECK ${ }^{\ddagger}$
}

\begin{abstract}
The dynamics of a closed loop thermosyphon are considered. The model assumes a prescribed heat flux along the loop wall and the contribution of axial diffusion. The well-posedness of the model which consists of a coupled ODE and PDE is shown for both the case with diffusion and without diffusion. Boundedness of solutions, the existence of an attractor, and an inertial manifold is proven, and an exact reduction to a low-dimensional model is obtained for the diffusion case. The reduced systems may have far fewer degrees of freedom than the reduction to the inertial manifold. For the three mode models, equivalence with the classical Lorenz equations is shown. Numerical results are presented for five mode models.
\end{abstract}

Key words. natural convection, asymptotic behavior, inertial manifold

AMS subject classifications. 35L50, 76E30

PII. S0036139996304184

1. Introduction. In this paper we present an analytical and numerical analysis of a class of models describing the motion of a fluid inside a closed loop circuit, driven by gravity, natural convection, pumps, etc. These types of devices are called thermosyphons and appear in numerous science and engineering fields. The first derivation of the basic equations of motion goes back to [11] and [20], after which several other generalizations, based on different physical assumptions, have been introduced; see, for example, [2], [5], and [7]. The monograph [10] contains a broad overview of the applications and the engineering interest in thermosyphons.

In all of these references, it is assumed that the loop is closed and completely full of fluid. The cross sectional area of the loop is assumed to be constant, and the velocity of the fluid is assumed to be independent of position in the circuit. This means that velocity $v(t)$ is assumed to be a scalar quantity depending only on time and satisfying an ODE. On the other hand, the other relevant quantity, namely, temperature, $T(t, x)$, is a function of time and position along the loop and satisfies a PDE.

In the absence of external pumping of the fluid, the mechanisms involved in the onset and sustainment of the fluid motion are the differences in temperature, the distribution of gravitational forces, the friction of the fluid at the inner wall of the loop, and transport of temperature, i.e. natural convection. However, most of these models neglect the contribution of axial heat diffusion. One of our goals in this paper is to study the influence of the diffusion mechanism on the dynamics and performance of thermosyphons.

*Received by the editors May 24, 1996; accepted for publication (in revised form) February 25, 1997; published electronically May 8, 1998. This research was partially supported by NATO Collaborative Research grant CRG-940655.

http://www.siam.org/journals/siap/58-4/30418.html

†Departamento de Matemática Aplicada, Universidad Complutense de Madrid, Madrid 28040, Spain (arober@sunma4.mat.ucm.es). The research of this author was partially supported by CICYT, PB93-0438, Spain, and EEC grant SC1-CT91-0732.

$\ddagger$ Department of Mathematical and Computer Sciences, Colorado School of Mines, Golden, CO 80401 (erikvv@poincare.mines.edu). The research of this author was partially supported by NSF grant DMS-9505049. 
Thermosyphon models differ due to differences in the geometry of the loop (which in most cases is assumed to be of simple type: either circular [5], [12] or consisting of straight vertical/horizontal branches joined together [2], [11], [20]), the friction law assumed at the inner wall of the circuit (linear in [5], [11], [20], quadratic in [2], [9], [12]), the distribution of external heat sources or sinks around the loop, and the heat flux the device exchanges with its surroundings. It is customary to take the prescribed flux case, $h=h(t, x)$ given [12], [9], or Newton's linear cooling law $h=k\left(T_{a}-T\right)$, where $k$ is a positive quantity, sometimes depending on the velocity, and $T_{a}(x)$ is the (given) ambient temperature distribution, [2], [20], or a blend of these two cases [5]. General geometries have been considered in [9] and [19], where the former considers a quadratic friction law and prescribed heat flux and the latter considers a general friction law and Newton's cooling law.

In this paper we study the model with axial diffusion and with a prescribed heat flux

$$
\left\{\begin{array}{l}
\frac{d v}{d t}+G(v) v=\oint T f, v(0)=v_{0} \\
\frac{\partial T}{\partial t}+v \frac{\partial T}{\partial x}=h(x)+\nu \frac{\partial^{2} T}{\partial x^{2}}, T(0, x)=T_{0}(x),
\end{array}\right.
$$

where we assume throughout that $G(v)$, which specifies the friction law at the inner wall of the loop, is positive and bounded away from zero; i.e., $G(v) \geq G_{0}>0$. It is usually taken to be $G(v)=G$, a positive constant for the linear friction case [11], $[20],[5]$, or $G(v)=|v|$ for the quadratic law [12], [9], or even a rather general function given by $G(v)=g(\operatorname{Re}|v|)|v|$, where $R e$ is a Reynolds-like number that is assumed to be large, and $g$ is a smooth strictly positive function defined on $(0, \infty)$ such that $g(s) \approx A / s$ as $s \rightarrow 0$ where $A$ is a positive constant and $g(s) \approx 1$ as $s \rightarrow \infty[19]$, [14], [16]. The parameter $\nu \geq 0$ denotes the diffusion coefficient, $x \in(0,1)$ is the arc length, and $\oint=\int_{0}^{1} d x$ denotes integration along the closed path of the circuit. The function $f=\frac{d z}{d x}$ represents the variation in height along the circuit, so $f$ describes the geometry of the loop and the distribution of gravitational forces. Note that $\oint f=0$. The function $h$ represents the heat transfer law across the loop wall. The functions $G, f$, and $h$ incorporate relevant physical constants of the model, such as the cross sectional area $D$, the length of the loop $L$, the Prandtl, Rayleigh, or Reynolds numbers, etc.

For (1.1) we obtain, for $T(t, x)=\sum_{k \in \mathbb{Z}^{*}} a_{k}(t) e^{2 \pi k i x}, h(x)=\sum_{k \in K} b_{k} e^{2 \pi k i x}$, and $f(x)=\sum_{k \in J} c_{k} e^{2 \pi k i x}$, where $K, J \subset \mathbb{Z}^{*}=\mathbb{Z} \backslash\{0\}$, a reduced system of the form

$$
\left\{\begin{array}{l}
\frac{d v}{d t}+G(v) v=\sum_{k \in K \cap J} a_{k}(t) \bar{c}_{k}, \\
\dot{a}_{k}(t)+\left(2 \pi i k v(t)+\nu 4 \pi^{2} k^{2}\right) a_{k}(t)=b_{k}, \quad k \in K \cap J
\end{array}\right.
$$

where $a_{-k}=\bar{a}_{k}, b_{-k}=\bar{b}_{k}$, and $c_{-k}=\bar{c}_{k}$.

We note that the reduced system here with diffusion resembles the reduced system obtained without diffusion in [14] and [16] for models of the form

$$
\left\{\begin{array}{l}
\frac{d v}{d t}+G(v) v=\oint T f \\
\frac{\partial T}{\partial t}+v \frac{\partial T}{\partial x}=H(v)\left(T_{a}-T\right)
\end{array}\right.
$$


with $f$ and $G$ as above and $H$ a positive function bounded away from zero. The model (1.3) corresponds to the one we consider in this paper in the case of no diffusion $\nu=0$, and a heat flux given by Newton's linear cooling law $h=H(v)\left(T_{a}-T\right)$ with $T_{a}$ the given ambient temperature. Assuming the same Fourier expansions as above for $T$ and $f$ and assuming $T_{a}(x)=\sum_{k \in K} b_{k} e^{2 \pi k i x}$, it is shown in [14] that the dynamics of the full system (1.3) is given by the reduced set of ODEs

$$
\left\{\begin{array}{l}
\frac{d v}{d t}+G(v) v=\sum_{k \in K \cap J} a_{k}(t) \bar{c}_{k}, \\
\dot{a}_{k}(t)+(2 \pi i k v(t)+H(v(t))) a_{k}(t)=H(v(t)) b_{k}, \quad k \in K \cap J .
\end{array}\right.
$$

Note the similarity between (1.2) and (1.4), despite the fact that from the physical viewpoint the dissipative mechanism in these two models is completely different, as are the equations (1.1) and (1.3) and their mathematical properties.

To understand this, note that the only nonlinearity in the temperature equations in (1.1) and (1.3) comes from the coupling with the scalar velocity equation through the transport term. Therefore, in many respects the equations behave as if they are linear, and any set of Fourier modes is an invariant set in phase space (modulo effects due to heating). As a result, there is no cascading of energy (as is the case for the Navier-Stokes equations), and linear damping is sufficient to ensure exponential decay at a uniform rate for those modes that are not driven. The dissipation due to the Laplacian term in (1.1) is not fundamentally stronger than linear damping, and hence one expects (1.1) and (1.3) to behave similarly. A similar argument was used for equations describing rotating elastic beams by Bloch and Titi in [1].

For (1.1) we present an analysis beginning with well-posedness and boundedness of solutions. For the case with diffusion $\nu>0$, the existence of an attractor and an inertial manifold is shown and an explicit reduction to low-dimensional systems is obtained. It is noteworthy that we are able to obtain an exact finite-dimensional reduction (1.2) that may have a much lower number of degrees of freedom than the reduction to the inertial manifold. This leads to the derivation of $2 n+1$ mode models for $n=1,2, \ldots$. In particular, the three-dimensional model is shown to contain the Lorenz system as a special case. This can be considered as an example of diffusion induced chaos since for ranges of the diffusion coefficient $\nu$ we obtain the Lorenz model in the "chaotic" parameter range. Numerical results are presented for five mode models, and Lorenz-type behavior is observed under realistic physical assumptions. The numerical results illustrate the dependence on the diffusion parameter $\nu$, and for different values of $\nu$ we observe convergence to equilibria, convergence to periodic solutions, as well as Lorenz-type behavior. On the other hand, in the case of no diffusion $\nu=0$, the existence of unbounded solutions is shown, although our numerical results suggest that stable bounded solutions do exist. In some cases reduction to lowdimensional systems is also possible for $\nu=0$. See [3] for other Lorenz models.

2. Well-posedness and boundedness. In this section we discuss the wellposedness and boundedness of solutions of the thermosyphon model (1.1). Observe that for $\nu \geq 0$ we have, integrating the equation for the temperature along the loop, $\frac{d}{d t} \oint T=\oint h$ and then $\oint T(t)=\oint T_{0}+t \oint h$. Therefore, the temperature is unbounded as $t \rightarrow \infty$, unless $\oint h=0$. However, taking $\theta=T-\oint T$ and $\hat{h}=h-\oint h$ reduces to the case $\oint T(t)=\oint T_{0}=\oint h=0$, since $\theta$ would satisfy

$$
\frac{\partial \theta}{\partial t}+v \frac{\partial \theta}{\partial x}=\hat{h}(x)+\nu \frac{\partial^{2} \theta}{\partial x^{2}}
$$


and $\oint T f=\oint \theta f$, since $\oint f=0$. Therefore, hereafter we consider the system (1.1) where all functions have zero average.

Also, if $\nu>0$ the operator $\nu A=-\nu \frac{\partial^{2}}{\partial x^{2}}$, together with periodic boundary conditions, is an unbounded, self-adjoint operator with compact resolvent in $L_{\text {per }}^{2}(0,1)$ that is positive when restricted to the space of zero average functions $\dot{L}_{\text {per }}^{2}(0,1)$. Hence the second equation in (1.1) is of parabolic type for $\nu>0$.

2.1. The case with diffusion: $\boldsymbol{\nu}>\mathbf{0}$. We write the system (1.1) as

$$
\frac{d}{d t}\left(\begin{array}{c}
v \\
T
\end{array}\right)+\left(\begin{array}{cc}
0 & 0 \\
0 & -\nu \frac{\partial^{2}}{\partial x^{2}}
\end{array}\right)\left(\begin{array}{c}
v \\
T
\end{array}\right)=\left(\begin{array}{l}
F_{1}(v, T) \\
F_{2}(v, T)
\end{array}\right)
$$

with $F_{1}(v, T)=-G(v) v+\oint T f$ and $F_{2}(v, T)=h(x)-v \frac{\partial T}{\partial x}$ and initial data $\left(\begin{array}{c}v \\ T\end{array}\right)(0)=$ $\left(\begin{array}{c}v_{0} \\ T_{0}\end{array}\right)$.

The operator

$$
B=\left(\begin{array}{cc}
0 & 0 \\
0 & -\nu \frac{\partial^{2}}{\partial x^{2}}
\end{array}\right)
$$

is a sectorial operator in $\mathbb{R} \times \dot{L}_{\text {per }}^{2}(0,1)$ with domain $D(B)=\mathbb{R} \times \dot{H}_{\text {per }}^{2}(0,1)$ and has compact resolvent, where

$$
\begin{gathered}
\dot{L}_{\text {per }}^{2}(0,1)=\left\{u \in L_{\text {loc }}^{2}(\mathbb{R}), u(x+1)=u(x) \text { a.e., } \oint u=0\right\}, \\
\dot{H}_{\text {per }}^{m}(0,1)=H_{\text {loc }}^{m}(\mathbb{R}) \cap \dot{L}_{\text {per }}^{2}(0,1) .
\end{gathered}
$$

The nonlinear terms satisfy $F_{1}: \mathbb{R} \times \dot{L}_{\text {per }}^{2}(0,1) \rightarrow \mathbb{R}, F_{2}: \mathbb{R} \times \dot{H}_{\text {per }}^{1}(0,1) \rightarrow$ $\dot{L}_{\text {per }}^{2}(0,1)$, and $F_{2}: \mathbb{R} \times \dot{L}_{\text {per }}^{2}(0,1) \rightarrow \dot{H}_{\text {per }}^{-1}(0,1)$ and are Lipschitz and bounded on bounded sets, provided $f, h \in \dot{L}_{\text {per }}^{2}(0,1)$.

Therefore, using the variations of constants formula for the system above, we look for solutions of the fixed point problem

$$
\begin{gathered}
v(t)=v_{0}-\int_{0}^{t} G(v(s)) v(s) d s+\int_{0}^{t}(\oint T(s) f) d s \\
T(t, x)=e^{-\nu A t} T_{0}(x)+\int_{0}^{t} e^{-\nu A(t-s)} h(x) d s-\int_{0}^{t} e^{-\nu A(t-s)} v(s) \frac{\partial T(s, x)}{\partial x} d s
\end{gathered}
$$

where $(v, T) \in C\left(\left[0, t_{0}\right], Y\right)$.

Then, using the results and techniques of [8], we obtain Theorem 2.1.

THEOREM 2.1. Assume that $G(v) v$ is locally Lipschitz, and that $f, h \in \dot{L}_{\text {per }}^{2}(0,1)$. Then for every $\left(v_{0}, T_{0}\right) \in \mathbb{R} \times \dot{L}_{\text {per }}^{2}(0,1)$ there exists a unique solution of $(1.1)$ satisfying

$$
\begin{gathered}
(v, T) \in C\left([0, \infty), \mathbb{R} \times \dot{L}_{\text {per }}^{2}(0,1)\right) \cap C\left((0, \infty), \mathbb{R} \times \dot{H}_{p e r}^{2}(0,1)\right), \\
\left(\dot{v}, \frac{\partial T}{\partial t}\right) \in C\left((0, \infty), \mathbb{R} \times \dot{H}_{p e r}^{2-\delta}(0,1)\right)
\end{gathered}
$$

for every $\delta>0$. In particular, (1.1) defines a nonlinear semigroup in $\mathbb{R} \times \dot{L}_{\text {per }}^{2}(0,1)$. 
Proof. Local existence and regularity follow easily from the variation of constants formula and [8]. For this, just note that the operator $B$, defined above, is also sectorial in $\mathbb{R} \times \dot{H}_{\text {per }}^{-1}(0,1)$ with domain $\mathbb{R} \times \dot{H}_{\text {per }}^{1}(0,1)$ and has compact resolvent. Note that in this context the operator $L=-\frac{\partial^{2}}{\partial x^{2}}$ must be understood in the variational sense; i.e., for every $T, \phi \in \dot{H}_{p e r}^{1}(0,1)$,

$$
\langle L(T), \phi\rangle=\oint \frac{\partial T}{\partial x} \frac{\partial \phi}{\partial x}
$$

and $\dot{L}_{\text {per }}^{2}(0,1)$ coincides with the fractional space of exponent $1 / 2$ [8].

Now using that $F_{2}: \mathbb{R} \times \dot{L}_{\text {per }}^{2}(0,1) \rightarrow \dot{H}_{\text {per }}^{-1}(0,1)$ is Lipschitz and bounded on bounded sets, we obtain local existence for initial data in $\mathbb{R} \times \dot{L}_{p e r}^{2}(0,1)$. In fact, from the smoothing effect of the equations, we have $(v, T) \in C\left([0, M), \mathbb{R} \times \dot{L}_{\text {per }}^{2}(0,1)\right) \cap$ $C\left((0, M), \mathbb{R} \times \dot{H}_{p e r}^{1}(0,1)\right)$ and $\left(\dot{v}, \frac{\partial T}{\partial t}\right) \in C\left((0, M), \mathbb{R} \times \dot{H}_{p e r}^{1-\delta}(0,1)\right)$ for some positive $M$ and any $\delta>0$. Now for $\epsilon>0$ small we have $(v(\epsilon), T(\epsilon)) \in \mathbb{R} \times \dot{H}_{p e r}^{1}(0,1)$ and, therefore, since $F_{2}: \mathbb{R} \times \dot{H}_{\text {per }}^{1}(0,1) \rightarrow \dot{L}_{\text {per }}^{2}(0,1)$ is also Lipschitz and bounded on bounded sets, we have $(v, T) \in C\left([\epsilon, M), \mathbb{R} \times \dot{H}_{\text {per }}^{1}(0,1)\right) \cap C\left((\epsilon, M), \mathbb{R} \times \dot{H}_{\text {per }}^{2}(0,1)\right)$ and $\left(\dot{v}, \frac{\partial T}{\partial t}\right) \in C\left((\epsilon, M), \mathbb{R} \times \dot{H}_{\text {per }}^{2-\delta}(0,1)\right)$. Since $\epsilon$ is arbitrary, we obtain the regularity of the local solution.

For global existence, we must show that solutions are bounded in the norm of $\mathbb{R} \times \dot{L}_{\text {per }}^{2}(0,1)$ on finite time intervals. For this, note that multiplying the equation for the temperature by $T$, integrating on $(0,1)$, and denoting by $\|\cdot\|$ the norm in $\dot{L}_{\text {per }}^{2}(0,1)$, we have

$$
\frac{1}{2} \frac{d}{d t}\|T\|^{2}+\nu\left\|\frac{\partial T}{\partial x}\right\|^{2}=\oint h T
$$

since $\oint T \frac{\partial T}{\partial x}=0$ due to the periodic boundary conditions. On the other hand, integrating the equation for the velocity, we have

$$
v(t)=v_{0} e^{-\int_{0}^{t} G(v)}+\int_{0}^{t}(\oint T f) e^{-\int_{s}^{t} G(v)} d s .
$$

Hence we find that $\|T(t)\|$ is bounded for finite time and so is $|v(t)|$, and hence we have a global solution and a nonlinear semigroup in $\mathbb{R} \times \dot{L}_{p e r}^{2}(0,1)$.

In order to obtain asymptotic bounds on the solutions as $t \rightarrow \infty$, we make use of the following result proven in [16].

LEMMA 2.2 (l'Hôpital's lemma). Assume $f$ and $g$ are real differentiable functions on $(a, b), b \leq \infty, g^{\prime}(x) \neq 0$ on $(a, b)$ and $\lim _{x \rightarrow b} g(x)=\infty$.

(i) If $\limsup _{x \rightarrow b} \frac{f^{\prime}(x)}{g^{\prime}(x)}=L$, then $\limsup _{x \rightarrow b} \frac{f(x)}{g(x)} \leq L$.

(ii) If $\liminf _{x \rightarrow b} \frac{f^{\prime}(x)}{g^{\prime}(x)}=L$, then $\liminf _{x \rightarrow b} \frac{f(x)}{g(x)} \geq L$.

With this, we have Theorem 2.2.

THEOREM 2.3. For any solution of (1.1) we have

$$
\begin{gathered}
\limsup _{t \rightarrow \infty}\|T\| \leq \frac{\|h\|}{\nu 4 \pi^{2}}, \\
\limsup _{t \rightarrow \infty}|v(t)| \leq \frac{\|h\|\|f\|}{\nu 4 \pi^{2}} \limsup _{t \rightarrow \infty} \frac{1}{G(v(t))} \leq \frac{\|h\|\|f\|}{\nu 4 \pi^{2} G_{0}} .
\end{gathered}
$$


Therefore, (1.1) has a global compact and connected attractor, $\mathcal{A}$, in $\mathbb{R} \times \dot{L}_{\text {per }}^{2}(0,1)$.

Proof. From (2.4), $\frac{1}{2} \frac{d}{d t}\|T\|^{2}+\nu\left\|\frac{\partial T}{\partial x}\right\|^{2}=\oint h T$. Using the Cauchy-Schwarz and Young inequalities and then the Poincaré inequality, since $\oint T=0$, we obtain

$$
\frac{d}{d t}\|T\|^{2}+\nu 4 \pi^{2}\|T\|^{2} \leq \frac{\|h\|^{2}}{\nu 4 \pi^{2}}
$$

and by elementary integration we obtain (2.6).

Now let $I(t)=\oint T(t) f$ so that $\limsup _{t \rightarrow \infty}|I(t)| \leq \frac{\|h\|\|f\|}{\nu 4 \pi^{2}}$. From $(2.5), v(t)=$ $v_{0} e^{-\int_{0}^{t} G(v)}+\int_{0}^{t} I(s) e^{-\int_{s}^{t} G(v)} d s$, and using l'Hôpital's lemma for the function $F(t)=$ $\int_{0}^{t} I(s) e^{-\int_{s}^{t} G(v)} d s=\left(\int_{0}^{t} I(s) e^{\int_{0}^{s} G(v)} d s\right) /\left(e^{\int_{0}^{t} G(v)}\right)$, we have $\lim \sup _{t \rightarrow \infty} F(t) \leq$ $\frac{\|h\|\|f\|}{\nu 4 \pi^{2}} \lim \sup _{t \rightarrow \infty} \frac{1}{G(v(t))}<\infty$ and we obtain $(2.7)$.

Since the sectorial operator $B$ defined above has compact resolvent the rest follows from [6, Theorems 4.2.2 and 3.4.8].

2.2. The case with no diffusion: $\boldsymbol{\nu}=\mathbf{0}$. The system now reads

$$
\left\{\begin{array}{l}
\frac{d v}{d t}+G(v) v=\oint T f, v(0)=v_{0} \\
\frac{\partial T}{\partial t}+v \frac{\partial T}{\partial x}=h(x), T(0, x)=T_{0}(x)
\end{array}\right.
$$

and therefore it is no longer of parabolic type.

To prove that the system is well posed, note that if $v(t)$ is a given continuous function then the second equation can be integrated along characteristics to obtain

$$
T(v)(t, x)=T_{0}\left(x-\int_{0}^{t} v\right)+\int_{0}^{t} h\left(x-\int_{s}^{t} v\right) d s
$$

and plugging this into the equation for the velocity yields

$$
\frac{d v}{d t}+G(v) v=\oint T(v) f, \quad v(0)=v_{0} .
$$

It remains to find a solution of this nonlocal differential equation. Note that for $T_{0}, h \in \dot{L}_{p e r}^{2}(0,1)$, and since in this space the translations are continuous isometries, (2.10) defines a continuous function of time with values in this space. Although we restrict ourselves to $\dot{L}_{\text {per }}^{2}(0,1)$, many other choices of space are possible for solving problem (2.9). In fact any Banach space of 1-periodic functions of $x$ having zero mean and in which translations are continuous isometries can be used as an "admissible space"; see [14]. Also note that the function given in (2.10) is an integral solution of the PDE, which is satisfied only if $T_{0}$ and $h$ are differentiable. In particular, if $T_{0}, h \in \dot{H}_{p e r}^{1}(0,1)$, then $T(v)$ is continuous with values in $\dot{H}_{p e r}^{1}(0,1)$ and satisfies the PDE in (2.9) as an equality in $\dot{L}_{\text {per }}^{2}(0,1)$ a.e. in time.

Then we have Theorem 2.3 .

THEOREM 2.4. Assume $G(v) v$ is locally Lipschitz and $T_{0}, f, h \in \dot{L}_{\text {per }}^{2}(0,1)$. Moreover, assume either $f \in \dot{H}_{\text {per }}^{1}(0,1)$ or $T_{0}, h \in \dot{H}_{p e r}^{1}(0,1)$ and $v_{0} \in \mathbb{R}$. Then there exists a unique solution of (2.9) satisfying

$$
(v, T) \in C\left([0, \infty), \mathbb{R} \times \dot{L}_{p e r}^{2}(0,1)\right),
$$


and $T$ satisfies the PDE in the sense of (2.10). If $T_{0}, h \in \dot{H}_{p e r}^{1}(0,1)$, then the solution is in fact in $C\left([0, \infty), \mathbb{R} \times \dot{H}_{p e r}^{1}(0,1)\right)$ and $\left(\dot{v}, \frac{\partial T}{\partial t}\right) \in C\left((0, \infty), \mathbb{R} \times \dot{L}_{\text {per }}^{2}(0,1)\right)$.

Proof. As noted above we need to solve the fixed point problem

$$
v(t)=\mathcal{F}(v)(t)=v_{0}-\int_{0}^{t} G(v(s)) v(s) d s+\int_{0}^{t}(\oint T(v)(s) f) d s
$$

on a space of continuous functions. More precisely, we take $W=\{v \in C[0, L], v(0)=$ $\left.v_{0},\left|v(t)-v_{0}\right| \leq M\right\}$, endowed with the sup norm, with $L$ and $M$ to be chosen, and prove that $\mathcal{F}$ is a contraction on $W$.

From the expression for $T(v)$ we have $\|T(v)(t)\| \leq\left\|T_{0}\right\|+t\|h\|$ and this, together with the local Lipschitz property of $G(v) v$, shows that for fixed $M, \mathcal{F}(W) \subset W$ if $L$ is sufficiently small.

To show that $\mathcal{F}$ is a contraction it is clear that we must prove some Lipschitz dependence on $\oint T(v) f$ with respect to $v \in W$.

First, assume that $T_{0}, h \in \dot{H}_{p e r}^{1}(0,1)$. Then verify that $\|T(\cdot+s)-T(\cdot)\| \leq c_{1}|s|$ for all $s \in \mathbb{R}$. If $v_{i} \in W$ for $i=1,2$, then

$$
\left|\oint\left(T_{0}\left(x-\int_{0}^{t} v_{1}\right)-T_{0}\left(x-\int_{0}^{t} v_{2}\right)\right) f(x) d x\right| \leq c_{1}\|f\| t \sup _{\left[0, t_{0}\right]}\left|v_{1}-v_{2}\right|
$$

and

$$
\left|\int_{0}^{t} \oint\left(h\left(x-\int_{s}^{t} v_{1}\right)-h\left(x-\int_{s}^{t} v_{2}\right)\right) f(x) d x d s\right| \leq c_{1}\|f\| t^{2} \sup _{\left[0, t_{0}\right]}\left|v_{1}-v_{2}\right| .
$$

With these we find that $\mathcal{F}$ is Lipschitz on $W$ with a Lipschitz constant depending on $L$ and $M$ that tends to zero as $L \rightarrow 0$ and then $\mathcal{F}$ is a contraction for small enough $L$.

If instead $f \in \dot{H}_{p e r}^{1}(0,1)$, then $\|f(\cdot+s)-f(\cdot)\| \leq c_{2}|s|$ for all $s \in \mathbb{R}$ and

$$
\begin{aligned}
\mid \oint( & \left.T_{0}\left(x-\int_{0}^{t} v_{1}\right)-T_{0}\left(x-\int_{0}^{t} v_{2}\right)\right) f(x) d x \mid \\
\quad & \left|\oint T_{0}(x)\left(f\left(x+\int_{0}^{t} v_{1}\right)-f\left(x+\int_{0}^{t} v_{2}\right)\right) d x\right| \\
& \leq c_{2}\left\|T_{0}\right\| t \sup _{\left[0, t_{0}\right]}\left|v_{1}-v_{2}\right|
\end{aligned}
$$

and

$$
\begin{gathered}
\left|\int_{0}^{t} \oint\left(h\left(x-\int_{s}^{t} v_{1}\right)-h\left(x-\int_{s}^{t} v_{2}\right)\right) f(x) d x d s\right| \\
=\left|\int_{0}^{t} \oint h(x)\left(f\left(x+\int_{s}^{t} v_{1}\right)-f\left(x+\int_{s}^{t} v_{2}\right)\right) d x d s\right| \leq c_{2}\|h\| t^{2} \sup _{\left[0, t_{0}\right]}\left|v_{1}-v_{2}\right|,
\end{gathered}
$$

and we obtain the same conclusion. Therefore, local well-posedness follows.

To prove global existence note that it is sufficient to prove that $v(t)$ is bounded on finite time intervals, since then from

$$
v(t)-v(r)=-\int_{r}^{t} G(v) v d s+\int_{r}^{t}(\oint T(v) f) d s
$$


we find that $v(t)$ is of Cauchy type as $t \rightarrow t_{0}$ for finite $t_{0}$. Consequently, the limit of $(v(t), T(t))$ as $t \rightarrow t_{0}$ exists in $\mathbb{R} \times \dot{L}_{\text {per }}^{2}(0,1)$ and the solution can be prolongated.

But again from (2.5), i.e., $v(t)=v_{0} e^{-\int_{0}^{t} G(v)}+\int_{0}^{t}(\oint T(v) f) e^{-\int_{s}^{t} G(v)} d s$, we obtain boundedness on finite time intervals and global existence follows.

As noted above, if $T_{0}, h \in \dot{H}_{p e r}^{1}(0,1)$ then $T$ satisfies the PDE in equation (2.9) as an equality in $\dot{L}_{p e r}^{2}(0,1)$ a.e in time. In particular, we have $\frac{\partial T}{\partial t} \in C((0, \infty)$, $\left.\dot{L}_{\text {per }}^{2}(0,1)\right)$.

The following result shows that in contrast to the case $\nu>0,(2.9)$ is not a dissipative system, since there may be unbounded solutions.

Proposition 2.5. Assume $\oint h f=0$ and $\oint T_{0} f=0$. Then the solution of (2.9) with initial data $\left(0, T_{0}\right)$ satisfies

$$
v(t)=0, T(t, x)=T_{0}(x)+t h(x) \text { for all } t \geq 0 .
$$

Proof. Note that multiplying the equation for the temperature by $f$ we have that $z(t)=\oint T(t) f$ and $v(t)$ are solutions of the nonautonomous system

$$
\begin{aligned}
& \dot{v}+G(v) v=z, v(0)=0 \\
& \dot{z}+m(t) v=0, z(0)=0
\end{aligned}
$$

where $m(t)=\oint \frac{\partial T(t)}{\partial x} f$. By uniqueness we obtain $v(t)=z(t)=0$, and then the equation for the temperature reads $\frac{\partial T}{\partial t}=h(x)$ and the rest is obvious.

3. Asymptotic behavior: Reduction to finite-dimensional systems. Consider the model (1.1) and the Fourier series expansions $T(t, x)=\sum_{k \in \mathbb{Z}^{*}} a_{k}(t) e^{2 \pi k i x}$, $h(x)=\sum_{k \in \mathbb{Z}^{*}} b_{k} e^{2 \pi k i x}$, and $f(x)=\sum_{k \in \mathbb{Z}^{*}} c_{k} e^{2 \pi k i x}$, where $\mathbb{Z}^{*}=\mathbb{Z} \backslash\{0\} ;$ then we easily find that for $k \in \mathbb{Z}^{*}, a_{k}(t)$ is a solution of

$$
\dot{a}_{k}(t)+\left(2 \pi i k v(t)+\nu 4 \pi^{2} k^{2}\right) a_{k}(t)=b_{k} .
$$

Note that since all functions involved are real, one has $a_{-k}=\bar{a}_{k}, b_{-k}=\bar{b}_{k}$, and $c_{-k}=\bar{c}_{k}$. Therefore, (1.1) is equivalent to the infinite system of ODEs consisting of (3.1) coupled with

$$
\frac{d v}{d t}+G(v) v=\sum_{k \in \mathbb{Z}^{*}} a_{k}(t) \bar{c}_{k} .
$$

These two equations reflect two of the main features of (1.1): the coupling between modes enters only through the velocity while diffusion acts as a linear damping term. In what follows we will exploit this explicit equation for the temperature modes to analyze the asymptotic behavior of the system and to obtain explicit low-dimensional models.

3.1. The case with diffusion: $\boldsymbol{\nu}>0$. We now consider the case $\nu>0$ and use inertial manifold techniques, in the spirit of [14], to give an explicit low-dimensional system of ODEs that describes the asymptotic dynamics of (1.1). The existence of an inertial manifold does not rely, in this case, on the existence of large gaps in the spectrum of the elliptic operator but on the invariance of certain sets of Fourier modes. A similar explicit construction was given by Bloch and Titi in [1] for a nonlinear beam equation where the nonlinearity occurs only through the appearance of the $L^{2}$ norm of the unknown. A related construction was given by Stuart in [18] for a nonlocal reaction-diffusion equation. 
3.1.1. Attractors and inertial manifolds. We first improve the bounds on the velocity and temperature of the previous section.

Proposition 3.1. For every solution of (1.1) we have

$$
\limsup _{t \rightarrow \infty}\left|a_{k}(t)\right| \leq \frac{\left|b_{k}\right|}{\nu 4 \pi^{2} k^{2}}
$$

and

$$
\limsup _{t \rightarrow \infty}|v(t)| \leq \limsup _{t \rightarrow \infty} \frac{I_{0}}{G(v(t))}<\infty,
$$

where $I_{0}:=\sum_{k \in \mathbb{Z}^{*}} \frac{\left|b_{k}\right|\left|c_{k}\right|}{\nu 4 \pi^{2} k^{2}}$.

Proof. The estimate (3.3) is easily obtained from equation (3.1), since $\left|a_{k}(t)\right| \leq$ $\left|a_{k}(0)\right| e^{-\nu 4 \pi^{2} k^{2} t}+\frac{\left|b_{k}\right|}{\nu 4 \pi^{2} k^{2}}\left(1-e^{-\nu 4 \pi^{2} k^{2} t}\right) \rightarrow \frac{\left|b_{k}\right|}{\nu 4 \pi^{2} k^{2}}$ as $t \rightarrow \infty$.

Now let $I(t)=\sum_{k \in \mathbb{Z}^{*}} a_{k}(t) \bar{c}_{k}$ so that $\limsup _{t \rightarrow \infty}|I(t)| \leq I_{0}$. From (2.5) we then have $v(t)=v_{0} e^{-\int_{0}^{t} G(v)}+\int_{0}^{t} I(s) e^{-\int_{s}^{t} G(v)} d s$. Applying l'Hôpital's lemma to

$$
F(t)=\int_{0}^{t} I(s) e^{-\int_{s}^{t} G(v)} d s=\frac{\int_{0}^{t} I(s) e^{\int_{0}^{s} G(v)} d s}{e^{\int_{0}^{t} G(v)}}
$$

implies $\lim \sup _{t \rightarrow \infty} F(t) \leq \lim \sup _{t \rightarrow \infty} \frac{I_{0}}{G(v(t))}<\infty$ and we obtain (3.4).

Note that the previous bound on the velocity is not well suited to the case in which $G(v)=g(\operatorname{Re}|v|)|v|$ since in this case the lower bound on $G$ (and therefore the upper bound on $v$ ) may depend on $R e$, for example, in the particular case $g(s)=1+\frac{A}{s}$, $G(v)=|v|+\frac{A}{R e} \geq \frac{A}{R e}$. To cover this case, we have Proposition 3.2.

Proposition 3.2. For any solution of (1.1) we have

$$
\limsup _{t \rightarrow \infty}|v(t)|^{2} \leq I_{0} \limsup _{t \rightarrow \infty} \frac{|v(t)|}{G(v(t))} .
$$

In particular, if $G(v)=g(\operatorname{Re}|v|)|v|$, then $\lim \sup _{t \rightarrow \infty} v^{2}(t) \leq \lim \sup _{t \rightarrow \infty} \frac{I_{0}}{g(\operatorname{Re}|v(t)|)} \leq$ $\frac{I_{0}}{g_{0}}$ where $g_{0}=\inf _{s}\{g(s)\}$.

Proof. Multiplying the equation for the velocity in (3.2) by $v$, we have $\frac{1}{2} \frac{d\left(v^{2}\right)}{d t}+$ $G(v) v^{2}=v I(t)$ and from this we obtain, as in $(2.5), v^{2}(t)=v_{0}^{2} e^{-2 \int_{0}^{t} G(v)}+\int_{0}^{t} 2 v(s)$ $\times I(s) e^{-2 \int_{s}^{t} G(v)} d s$. Therefore, from l'Hôpital's lemma we find

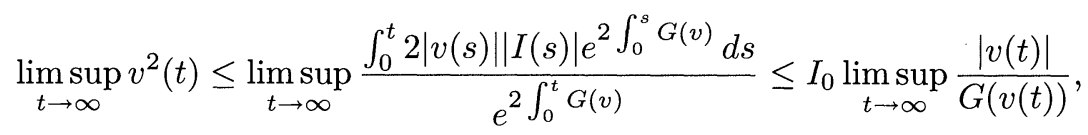

and the rest is obvious.

As a consequence, we have the following result on the smoothness of the attractor of (1.1). then

COROLlaRY 3.3. With the above assumptions, if $h \in \dot{H}_{\text {per }}^{m}(0,1)$, for some $m \geq 0$,

$$
\mathcal{A} \subset \mathbb{R} \times \dot{H}_{\text {per }}^{m+2}(0,1)
$$

and $\mathcal{A}$ is a compact set in that space. 
Proof. From equation (3.3), for any $(v, T) \in \mathcal{A}$ we have $\nu 4 \pi^{2} k^{2}\left|a_{k}\right| \leq\left|b_{k}\right|$. Since $\sum_{k \in \mathbb{Z}^{*}}|k|^{2 m}\left|b_{k}\right|^{2}<\infty, T \in \dot{H}_{\text {per }}^{m+2}(0,1)$ and $\mathcal{A} \subset[-M, M] \times \mathcal{B}$, where $M$ is an upper bound for the velocity, as in (2.7), (3.4), (3.5), and $\mathcal{B}=\left\{T \in \dot{H}_{\text {per }}^{m+2}(0,1), \nu 4 \pi^{2} k^{2}\left|a_{k}\right| \leq\right.$ $\left.\left|b_{k}\right|\right\}$. But the set $\mathcal{B}$ is compact in $\dot{H}_{\text {per }}^{m+2}(0,1)$ since for any sequence $\left\{T^{n}\right\}$ in $\mathcal{B}$ we can extract a subsequence that we still denote $\left\{T^{n}\right\}$ such that it converges weakly to a function $T$ and such that, for any $k \in \mathbb{Z}^{*}$, the Fourier coefficients verify $a_{k}^{n} \rightarrow a_{k}$ as $n \rightarrow \infty$, where $a_{k}$ is the $k$ th Fourier coefficient of $T$. Therefore, $\nu 4 \pi^{2} k^{2}\left|a_{k}\right| \leq\left|b_{k}\right|$ and for every integer $M$,

$$
\left\|T^{n}-T\right\|_{m+2}^{2} \leq \sum_{|k|=1}^{M}|k|^{2 m+4}\left|a_{k}^{n}-a_{k}\right|^{2}+C \sum_{|k|=M+1}^{\infty}|k|^{2 m}\left|b_{k}\right|^{2},
$$

where $\|\cdot\|_{m+2}$ denotes the norm in $\dot{H}_{p e r}^{m+2}(0,1)$. Hence the first term goes to zero as $n \rightarrow \infty$ and the second can be made arbitrarily small as $M \rightarrow \infty$. Consequently, $T \in \mathcal{B}$ and $T^{n} \rightarrow T$ in $\dot{H}_{p e r}^{m+2}(0,1)$ and the result is proved.

Note that this result reveals in particular the asymptotic smoothing of (1.1). In the next result we will prove that the dynamical system induced by (1.1) in $\mathbb{R} \times$ $\dot{L}_{\text {per }}^{2}(0,1)$ has an inertial manifold. According to [4] we have the following definition.

DEFINITION 3.4. Let $S(t), t \geq 0$, be a nonlinear semigroup in a Banach space $Y$ that has a global attractor $\mathcal{A}$. Then a smooth manifold $\mathcal{M} \subset Y$ is called an inertial manifold if

(i) $\mathcal{M}$ is positively invariant, i.e., $S(t) \mathcal{M} \subset \mathcal{M}$ for every $t \geq 0$;

(ii) $\mathcal{M}$ contains the attractor, i.e., $\mathcal{A} \subset \mathcal{M}$;

(iii) $\mathcal{M}$ is exponentially attracting in the sense that there exists a constant $M>0$ such that for every bounded set $B \subset Y$ there exists a constant $C=C(B)$ such that

$$
\operatorname{dist}(S(t) B, \mathcal{M}) \leq C e^{-M t}
$$

for every $t \geq 0$.

See, for example, [4], [15].

Assume now the heat flux is given by

$$
h(x)=\sum_{k \in K} b_{k} e^{2 \pi k i x} \in \dot{L}_{p e r}^{2}(0,1),
$$

where $K \subset \mathbb{Z}^{*}$. Then denote by $V$ the closed linear subspace of $\dot{L}_{\text {per }}^{2}(0,1)$ spanned by $\left\{e^{2 \pi k i x}, k \in K\right\}$, and consider the following spectral decomposition in $\dot{L}_{\text {per }}^{2}(0,1)$ : $T=T_{1}+T_{2}$, where $T_{1}$ denotes the projection of $T$ onto $V$ and $T_{2}$ the projection onto the space generated by $\left\{e^{2 \pi k i x}, k \notin K\right\}$. Note that $(1.1)$ is equivalent to

$$
\left\{\begin{array}{l}
\frac{d v}{d t}+G(v) v=\oint\left(T_{1}+T_{2}\right) f, v(0)=v_{0} \\
\frac{\partial T_{1}}{\partial t}+v \frac{\partial T_{1}}{\partial x}=h(x)+\nu \frac{\partial^{2} T_{1}}{\partial x^{2}}, T_{1}(0)=T_{01} \\
\frac{\partial T_{2}}{\partial t}+v \frac{\partial T_{2}}{\partial x}=\nu \frac{\partial^{2} T_{2}}{\partial x^{2}}, T_{2}(0)=T_{02} .
\end{array}\right.
$$

Note that from (3.1) if $b_{k}=0$ then the $k$ th mode for the temperature is damped out exponentially and therefore the space $V$ attracts the dynamics for the temperature. This is precisely stated in the following result. 
THEOREM 3.5. With the notation above, $\mathcal{M}=\mathbb{R} \times V$ is an inertial manifold for the flow of (1.1) in $\mathbb{R} \times \dot{L}_{\text {per }}^{2}(0,1)$.

Moreover, if $f \in V$ the inertial manifold $\mathcal{M}$ has the exponential tracking property; that is, for every $\left(v_{0}, T_{0}\right) \in \mathbb{R} \times \dot{L}_{\text {per }}^{2}(0,1)$ there exists $\left(v_{1}, T_{1}\right) \in \mathcal{M}$ such that if $\left(v_{i}(t), T_{i}(t)\right), i=0,1$, are the corresponding solutions of $(1.1)$, then $\left(v_{0}(t), T_{0}(t)\right)-$ $\left(v_{1}(t), T_{1}(t)\right) \rightarrow 0$ in $\mathbb{R} \times \dot{L}_{\text {per }}^{2}(0,1)$, at an exponential rate, as $t \rightarrow \infty$.

Proof. Since the third equation in (3.6) is linear in $T_{2}$, if $T_{02}(x)=0$, then $T_{2}(t, x)=0$ for every $t$; i.e., $\mathcal{M}$ is an invariant manifold. Now, observe that

$$
\operatorname{dist}_{Y}((v(t), T(t)), \mathcal{M})=\operatorname{dist}_{\dot{L}_{p e r}^{2}}(T(t), V)=\left\|T_{2}(t)\right\| \leq\left\|T_{02}\right\| e^{-\nu 4 \pi^{2} t},
$$

and the manifold is exponentially attracting. Note that the last inequality comes from (2.4), with $h=0$, and the Poincaré inequality.

To prove the exponential tracking property just note that the flow inside $\mathcal{M}$ is given by setting $T_{2}=0$ in (3.6), i.e.,

$$
\left\{\begin{array}{l}
\frac{d v}{d t}+G(v) v=\oint T_{1} f \\
\frac{\partial T_{1}}{\partial t}+v \frac{\partial T_{1}}{\partial x}=h(x)+\nu \frac{\partial^{2} T_{1}}{\partial x^{2}} \\
T_{2}=0 .
\end{array}\right.
$$

Therefore, if $f \in V$, then $\oint T f=\oint T_{1} f$ and then if $(v(t), T(t)) \in \mathbb{R} \times \dot{L}_{\text {per }}^{2}(0,1)$ is a solution of (3.6), then $\left(v(t), T_{1}(t)\right) \in \mathcal{M}$ and it is still a solution of (3.7). Hence $(v(t), T(t))-\left(v(t), T_{1}(t)\right)=\left(0, T_{2}(t)\right)$ and the right-hand side is of order $e^{-\nu 4 \pi^{2} t}$. Thus the theorem is proved.

In particular, note that if the set $K$ is finite then the inertial manifold $\mathcal{M}$ is of finite dimension and then (3.7) is equivalent to the system of ODEs

$$
\left\{\begin{array}{l}
\frac{d v}{d t}+G(v) v=\sum_{k \in K} a_{k}(t) \bar{c}_{k}, \\
\dot{a} k(t)+\left(2 \pi i k v(t)+\nu 4 \pi^{2} k^{2}\right) a_{k}(t)=b_{k}, k \in K .
\end{array}\right.
$$

3.1.2. The reduced subsystem. Assume now that the heat flux and the geometry of the loop are given, respectively, by

$$
h(x)=\sum_{k \in K} b_{k} e^{2 \pi k i x}, K \subset \mathbb{Z}^{*}, \quad \text { and } \quad f(x)=\sum_{k \in J} c_{k} e^{2 \pi k i x}, J \subset \mathbb{Z}^{*} .
$$

In view of (3.1) and (3.2) it is clear that the modes in $K \cap J$ will play an essential role in the dynamics as we now show. With the above notation, we further decompose $T_{1}$ as follows:

$$
T_{1}=\tau+\theta,
$$

where $\tau$ is the projection onto the space generated by $\left\{e^{2 \pi k i x}, k \in K \cap J\right\}$ and $\theta$ is the projection onto the space generated by $\left\{e^{2 \pi k i x}, k \in K \backslash J\right\}$. Finally, denote by $P$ the projection $P(v, T)=(v, \tau)$ and $Q=I-P$. With this notation, and decomposing 
$h$, as $h=\tau_{h}+\theta_{h},(1.1)$ and (3.6) can be decomposed as a system of the form

$$
\left\{\begin{array}{l}
\frac{d v}{d t}+G(v) v=\oint\left(\tau+T_{2}\right) f, v(0)=v_{0} \\
\frac{\partial \tau}{\partial t}+v \frac{\partial \tau}{\partial x}=\tau_{h}(x)+\nu \frac{\partial^{2} \tau}{\partial x^{2}}, \tau(0)=\left(T_{0}\right)_{\tau} \\
\frac{\partial \theta}{\partial t}+v \frac{\partial \theta}{\partial x}=\theta_{h}(x)+\nu \frac{\partial^{2} \theta}{\partial x^{2}}, \theta(0)=\left(T_{0}\right)_{\theta} \\
\frac{\partial T_{2}}{\partial t}+v \frac{\partial T_{2}}{\partial x}=\nu \frac{\partial^{2} T_{2}}{\partial x^{2}}, T_{2}(0)=T_{02}
\end{array}\right.
$$

since $\oint \theta f=0$. Note that, when setting $T_{2}=0$, the first three equations give the flow inside the inertial manifold $\mathcal{M}$, i.e., they are equivalent to (3.7), while the first two are the only nonlinearly coupled equations. Therefore, once this subsystem is solved the other unknowns are determined through linear nonhomogeneous equations.

To make this idea more precise in terms of semigroups and attractors, we proceed as follows. We denote by $S(t)$ the semigroup generated by (1.1) on $Y \stackrel{\text { def }}{=} \mathbb{R} \times \dot{L}_{\text {per }}^{2}(0,1)$ and by $S_{I}(t)$ its restriction to the inertial manifold $\mathcal{M}$, i.e., the semigroup generated by (3.7). We will find below a reduced semigroup on the reduced space $Y_{R} \stackrel{\text { def }}{=} P(Y)$, denoted $S_{R}(t)$, that, in a sense, determines the asymptotic behavior of $S_{I}(t)$ and therefore that of $S(t)$. Note that $S(t)$ and $S_{I}(t)$ have the same attractor, while the dimension of the space $Y_{R}$ might be much smaller than that of $\mathcal{M}$. The next result states, in particular, that the attractor of the full system can be reconstructed from the attractor of the reduced one.

THEOREM 3.6. With the notation above we have the following conditions.

(i) The system of equations

$$
\left\{\begin{array}{l}
\frac{d v}{d t}+G(v) v=\oint \tau f, v(0)=v_{0}, \\
\frac{\partial \tau}{\partial t}+v \frac{\partial \tau}{\partial x}=\tau_{h}(x)+\nu \frac{\partial^{2} \tau}{\partial x^{2}}, \tau(0)=\tau_{0}
\end{array}\right.
$$

defines a nonlinear semigroup, denoted $S_{R}(t)$, on $Y_{R}=P(Y)$ that can be identified with $P S_{I}(t) P=P S_{I}(t)$ restricted to $Y_{R}$.

(ii) If $\mathcal{A}$ denotes the maximal attractor of $(1.1)$, then $\mathcal{A}_{R}=P(\mathcal{A})$ is the maximal attractor of (3.9). Moreover

$$
\mathcal{A}=\mathcal{G}\left(\mathcal{A}_{R}\right)
$$

where $\mathcal{G}: \mathcal{A}_{R} \rightarrow \mathcal{A}$ is continuous.

(iii) If the set $K \cap J$ is finite, (3.9) is equivalent to a system of complex ODEs of the form (1.2). Consequently, the asymptotic behavior of (1.1) is described by an explicit system of ODEs in $\mathbb{R}^{N}$ with $N=|K \cap J|+1$ an odd number.

Proof.

(i) The proof of the existence and uniqueness of globally defined solutions for (3.9) follows the same lines as for (1.1). Therefore, the semigroup $S_{R}(t)$ is well defined. On the other hand, from (3.8) it is clear that the projection onto $Y_{R}$ of any solution of this system lying in the inertial manifold $\mathcal{M}$, i.e., with $T_{2}=0$, is a solution of $(3.9)$; that is, $S_{R}(t)=P S_{I}(t)$. At the same time, it is also clear that $P S_{I}(t)=P S_{I}(t) P$. Note that, however, $Y_{R}$ is not invariant for $S_{I}(t)$.

(ii) The existence of the attractor for $S_{R}(t)$ is the same as for (1.1). Therefore, $\mathcal{A}_{R}$ is well defined. Moreover, since $S_{R}(t)=P S_{I}(t) P=P S_{I}(t)$ on $Y_{R}$, and $S_{I}(t) \mathcal{A}=\mathcal{A}$, 
$P(\mathcal{A})$ is compact and invariant for $S_{R}(t)$; i.e., $S_{R}(t) P(\mathcal{A})=P(\mathcal{A})$. To see this, just note that

$$
S_{R}(t) P(\mathcal{A})=P S_{I}(t) P(\mathcal{A})=P S_{I}(t)(\mathcal{A})=P(\mathcal{A}) .
$$

Since $\mathcal{A}_{R}$ is the maximal bounded invariant set for $S_{R}(t)[6]$, we obtain $P(\mathcal{A}) \subset \mathcal{A}_{R}$.

Conversely, if $\left(v_{0}, \tau_{0}\right) \in \mathcal{A}_{R}$, we show below that there exists a unique $\theta_{0}$ such that $\left(v_{0}, \tau_{0}+\theta_{0}\right) \in \mathcal{A}$. That would prove that $\mathcal{A}_{R} \subset P(\mathcal{A})$. While proving this, we will actually show that $\theta_{0}=\mathcal{F}\left(v_{0}, \tau_{0}\right)$, where $\mathcal{F}$ is a continuous function. With this, we will have proved that $\mathcal{A}=\mathcal{G}\left(\mathcal{A}_{R}\right)$ with $\mathcal{G}=\left(I_{d}, \mathcal{F}\right)$ and $\mathcal{G}$ continuous. Therefore, the attractor of the full system $\mathcal{A}$ can be reconstructed from the attractor of the reduced one $\mathcal{A}_{R}$.

So, if $\theta_{0}$ is such that $\left(v_{0}, \tau_{0}+\theta_{0}\right) \in \mathcal{A}$, then the solution of (1.1) is defined for $t \in \mathbb{R}$ and then from the equation for $\theta$ in (3.8) and for $t>s$ we have

$$
\theta(t, x)=U(t, s) \theta(s, x)+\int_{s}^{t} U(t, z) \theta_{h}(x) d z,
$$

where $U(t, s)$ is the evolution operator generated by the solutions of the initial value problem, at time $s$, for the time dependent equation $\frac{\partial \theta}{\partial t}+v(t) \frac{\partial \theta}{\partial x}=\nu \frac{\partial^{2} \theta}{\partial x^{2}}$; see Lemma 3.1 below.

Since the solution is on the attractor, the $\theta$ component is bounded and then setting $t=0$ and letting $s \rightarrow-\infty$, we find

$$
\theta_{0}=\int_{-\infty}^{0} U(0, z) \theta_{h} d z=\mathcal{F}\left(v_{0}, \tau_{0}\right),
$$

which is well defined, since by Lemma 3.1 the integral of the norm converges.

Conversely, it is easy to check that the solution of (3.8) starting at $\left(v_{0}, \tau_{0}, \theta_{0}, 0\right)$ actually exists globally and is bounded. Hence it lies on the attractor $\mathcal{A}$. Note that the $\theta$ component is given by

$$
\theta(t, x)=\int_{-\infty}^{t} U(t, z) \theta_{h}(x) d z, \quad t \in \mathbb{R} .
$$

It just remains to prove that $\mathcal{F}$ is continuous. To this end, we denote, for $M>0$,

$$
\mathcal{F}_{M}\left(v_{0}, \tau_{0}\right)=\int_{-M}^{0} U(0, z) \theta_{h} d z .
$$

We will prove in the lemmas below that $\mathcal{F}_{M}$ is continuous. Assuming this, for a moment, we can prove that $\mathcal{F}_{M} \rightarrow \mathcal{F}$ uniformly on $\mathcal{A}_{R}$, and this proves that $\mathcal{F}$ is continuous. In fact, we have $\mathcal{F}\left(v_{0}, \tau_{0}\right)-\mathcal{F}_{M}\left(v_{0}, \tau_{0}\right)=\int_{-\infty}^{-M} U(0, z) \theta_{h}(x) d z$ so the right-hand side goes to zero uniformly in $\left(v_{0}, \tau_{0}\right)$ as $M$ goes to $\infty$.

(iii) The proof is obvious. Just note that $K \cap J$ is a symmetric set and $0 \notin K \cap J$, so $|K \cap J|$ is even. Also, the equations for $a_{k}$ and $a_{-k}=\bar{a}_{k}$ are conjugate, so when employing real variables, $a_{k}=x_{k}+i y_{k}$, we have a system in $\mathbb{R}^{N}$ with $N=|K \cap J|+1$ odd.

Note that given a heat flux term $h$, or geometry $f$, one can design the other such that $K \cap J$ has any predefined even number of elements. Therefore, one can design geometries or fluxes in such a way that (1.2) has any desired odd number of variables. 
Also note that the set $K \cap J$ can be much smaller than the set $K$ and therefore the reduced subsystem may possess far fewer degrees of freedom than the system on the inertial manifold. At the same time, observe that if $f$ or $h$ is perturbed such that one adds a new pair of conjugates modes to the set $K \cap J$, i.e., we pass from an $n$ mode model to an $n+2$ mode model, the former set of equations is included in the latter, and this shows the increasing complexity of the dynamics of system (1.2) as the number of elements in $K \cap J$ increases.

The simplest situation is described next.

Corollary 3.7. Assume $K \cap J=\emptyset$; then the attractor of (1.1) reduces to the point $\left(0, \theta_{\infty}(x)\right)$, where $\theta_{\infty}(x)$ is the unique solution in $\dot{H}_{\text {per }}^{2}(0,1)$ of

$$
\nu \frac{\partial^{2} \theta}{\partial x^{2}}=h(x)
$$

Proof. If $K \cap J=\emptyset$, then in (3.8) $\tau=0, \theta=T_{1}$, and $T_{2} \rightarrow 0$ exponentially. Since $G$ is strictly bounded away from zero it follows that $v(t) \rightarrow 0$ exponentially and then the function $\hat{\theta}=\theta-\theta_{\infty}$ satisfies the equation

$$
\frac{\partial \hat{\theta}}{\partial t}+v(t) \frac{\partial \hat{\theta}}{\partial x}=\nu \frac{\partial^{2} \hat{\theta}}{\partial x^{2}}-v(t) \frac{\partial \theta_{\infty}}{\partial x} .
$$

As for (2.4) we have

$$
\frac{1}{2} \frac{d}{d t}\|\hat{\theta}\|^{2}+\nu\left\|\frac{\partial \hat{\theta}}{\partial x}\right\|^{2}=-v(t) \oint \frac{\partial \theta_{\infty}}{\partial x} \hat{\theta}
$$

and proceeding as in (2.8) and using $v(t) \rightarrow 0$ we prove $\hat{\theta}(t) \rightarrow 0$.

Observe that this result implies in particular that the regularity of the attractor obtained in Corollary 3.1 is optimal. On the other hand, note that the modes corresponding to $k \notin K \cap J$ are determined as solutions of the linear nonhomogeneous equations

$$
\dot{a}_{k}(t)+\left(2 \pi i k v(t)+\nu 4 \pi^{2} k^{2}\right) a_{k}(t)=b_{k}, \quad k \notin K \cap J
$$

with initial data $a_{k}(0) \in \mathbb{C}$. Therefore, we call these the slave modes. Observe that solving (3.12) is equivalent to solving the equations for $\theta$ and $T_{2}$ in (3.8).

Now we prove the technical results needed in the proof of Theorem 3.2.

LEMMA 3.8. Let $v \in C^{1}(\mathbb{R})$ be given, and consider the initial value problem

$$
\frac{\partial \theta}{\partial t}+v(t) \frac{\partial \theta}{\partial x}=\nu \frac{\partial^{2} \theta}{\partial x^{2}}, \quad \theta(s)=\theta_{0}
$$

with $t \geq s$.

(i) Then the initial value problem defines a family of evolution operators $U(t, s)$, where $\theta(t)=U(t, s) \theta_{0}$, that satisfies

$$
\|U(t, s)\| \leq e^{-\nu 4 \pi^{2}(t-s)} \quad \text { for } t \geq s .
$$

(ii) If $\theta_{h}$ is a fixed function, then the unique solution of

$$
\frac{\partial \theta}{\partial t}+v(t) \frac{\partial \theta}{\partial x}=\theta_{h}(x)+\nu \frac{\partial^{2} \theta}{\partial x^{2}}, \quad \theta(s)=\theta_{0}
$$


with $t \geq s$, is given by

$$
\theta(t, x)=U(t, s) \theta_{0}(x)+\int_{s}^{t} U(t, z) \theta_{h}(x) d z
$$

Proof. The construction of $U(t, s)$ follows from [8, Theorem 7.1.3], since the time dependent part $B(t) \theta=v(t) \frac{\partial \theta}{\partial x}$ is Lipschitz continuous in time with values in $\mathcal{L}\left(X^{1 / 2}, X\right)$. The reader is referred to this result for several other properties of the family $U(t, s)$.

The estimate on the norm of $U(t, s)$ follows from (2.4), with $h=0$, and the Poincaré inequality, while the second part of the result is just a particular case of $[8$, Theorem 7.1.4].

LEMMA 3.9. The function $\mathcal{F}_{M}$ is continuous on $\mathcal{A}_{R}$.

Proof. Recalling the definition of $\mathcal{F}_{M}$, let $\left(v_{0, i}, \tau_{0, i}\right) \in \mathcal{A}_{R}$, for $i=1,2$, and consider the corresponding solutions of (3.9), denoting by $\theta_{i}(t, x)$ the solution of

$$
\frac{\partial \theta}{\partial t}+v_{i}(t) \frac{\partial \theta}{\partial x}=\nu \frac{\partial^{2} \theta}{\partial x^{2}}, \quad \theta\left(t_{0}\right)=\theta_{h}
$$

with $0 \geq t>t_{0} \geq-M$. Set $W=\theta_{1}-\theta_{2}$; then $W$ satisfies $W\left(t_{0}, x\right)=0$ and

$$
\frac{\partial W}{\partial t}+v_{1}(t) \frac{\partial W}{\partial x}+\left(v_{1}(t)-v_{2}(t)\right) \frac{\partial \theta_{2}}{\partial x}=\nu \frac{\partial^{2} W}{\partial x^{2}}
$$

Multiplying by $W$ and integrating in $x$, we obtain

$$
\frac{1}{2} \frac{d}{d t}\|W\|^{2}+\nu\left\|\frac{\partial W}{\partial x}\right\|^{2}=\left(v_{2}(t)-v_{1}(t)\right) \oint \frac{\partial \theta_{2}}{\partial x} W=\left(v_{1}(t)-v_{2}(t)\right) \oint \theta_{2} \frac{\partial W}{\partial x} .
$$

From the previous lemma, $\left\|\theta_{2}\right\| \leq e^{-\nu 4 \pi^{2}\left(t-t_{0}\right)}\left\|\theta_{h}\right\| \leq\left\|\theta_{h}\right\|$, and using Young's inequality and the Poincaré inequality we have

$$
\frac{d}{d t}\|W\|^{2}+4 \pi^{2} \nu\|W\|^{2} \leq \frac{\left\|\theta_{h}\right\|}{\nu}\left|v_{1}(t)-v_{2}(t)\right|^{2},
$$

and integrating on $\left(t_{0}, 0\right)$ we obtain

$$
\|W(0)\|^{2} \leq \frac{\left\|\theta_{h}\right\|}{\nu} \int_{t_{0}}^{0}\left|v_{1}(r)-v_{2}(r)\right|^{2} e^{4 \pi^{2} \nu r} d r
$$

and $W(0)=U_{1}\left(0, t_{0}\right) \theta_{h}-U_{2}\left(0, t_{0}\right) \theta_{h}$.

But then [8, Theorem 3.4.1] gives the continuous dependence of the mapping $\left(v_{0}, \tau_{0}(x)\right) \mapsto(v(t), \tau(t, x))$ on compact time intervals, and this shows that $\mathcal{F}_{M}\left(v_{0,1}, \tau_{0,1}\right)-$ $\mathcal{F}_{M}\left(v_{0,2}, \tau_{0,2}\right)$ is small if $v_{0,1}-v_{0,2}$ and $\tau_{0,1}(x)-\tau_{0,2}(x)$ are small.

3.1.3. The three-dimensional model: Lorenz chaos. Now we assume $K \cap$ $J=\{ \pm k\}$ and that $G$ is constant. Recalling (1.2) in this case and using the fact that the equation for $-k$ is conjugate to that for $k$, we have

$$
\begin{aligned}
& \frac{d v}{d t}+G v=2 \operatorname{Re}\left(a_{k}(t) \bar{c}_{k}\right), \\
& \dot{a}_{k}(t)+\left(2 \pi i k v(t)+\nu 4 \pi^{2} k^{2}\right) a_{k}(t)=b_{k} .
\end{aligned}
$$


First, by changing variables as $a_{k} \rightarrow a_{k} \bar{c}_{k} \frac{1}{\lambda}$ and $b_{k} \rightarrow b_{k} \bar{c}_{k} \frac{1}{\lambda}$, where $\lambda$ is a real parameter to be chosen below, we find

$$
\begin{aligned}
& \frac{d v}{d t}+G v=2 \lambda \operatorname{Re}\left(a_{k}(t)\right) \\
& \dot{a}_{k}(t)+\left(2 \pi i k v(t)+\nu 4 \pi^{2} k^{2}\right) a_{k}(t)=b_{k} .
\end{aligned}
$$

Taking real and imaginary parts and denoting $a_{k}(t)=x(t)+i y(t)$ and $b_{k}=A+i B$, we have

$$
\begin{gathered}
v^{\prime}+G v-2 \lambda x=0, \\
x^{\prime}+\nu 4 \pi^{2} k^{2} x-2 \pi k v y=A, \\
y^{\prime}+2 \pi k v x+\nu 4 \pi^{2} k^{2} y=B .
\end{gathered}
$$

Now changing $y \rightarrow B-y$, rescaling $v$ as $v \rightarrow 2 \pi k v$, and choosing $\lambda=\frac{G}{4 \pi k}$, we obtain

$$
\begin{gathered}
v^{\prime}+G v-G x=0, \\
x^{\prime}+\nu 4 \pi^{2} k^{2} x-B v+v y=A, \\
y^{\prime}+\nu 4 \pi^{2} k^{2} y-v x=0,
\end{gathered}
$$

which for the case $A=0$ corresponds precisely to the standard Lorenz system [13], [17] with parameters $\sigma=G, b=\nu 4 \pi^{2} k^{2}$, and $r=B$, except for the extra $\nu 4 \pi^{2} k^{2}$ in the second equation that is equal to one in the Lorenz system. Note that this coefficient could be set to one by a suitable time rescaling. For $\sigma>b+1$ and $r$ larger than some critical value $r_{h}$, see [17], the Lorenz equations are known to possess complicated dynamics. All of these behaviors are translated to the three-dimensional model of the thermosyphon. Note that the number $k$ plays a role in the dissipative part of the equations; thus for larger $k$, larger friction and larger $B$ are needed to enter the chaotic regime of the Lorenz system. Our numerical experiments described in the next section show that the same kind of behavior is actually present when $G$ is a nonlinear function of the velocity.

3.2. The case with no diffusion: $\boldsymbol{\nu}=\mathbf{0}$. Now we consider the case with no diffusion, and although we showed the system is not dissipative, we can, however, reduce it to a finite-dimensional system in several situations.

First note that when $\nu=0,(3.1)$ and (3.2) read

$$
\begin{gathered}
\frac{d v}{d t}+G(v) v=\sum_{k \in \mathbb{Z}^{*}} a_{k}(t) \bar{c}_{k}, \\
\dot{a}_{k}(t)+2 \pi i k v(t) a_{k}(t)=b_{k}, \quad k \in \mathbb{Z}^{*} .
\end{gathered}
$$

Assume now that the geometry of the loop is given by

$$
f(x)=\sum_{k \in J} c_{k} e^{2 \pi k i x}, \quad J \subset \mathbb{Z}^{*},
$$

and observe that only the modes in the set $J$ actually appear in the equation for the velocity above. This motivates us to consider the projection of a function $T \in$ $\dot{L}_{\text {per }}^{2}(0,1)$ onto the closed linear subspace of $\dot{L}_{\text {per }}^{2}(0,1)$ spanned by $\left\{e^{2 \pi k i x}, k \in J\right\}$, denoted $T_{J}$, and write $T=T_{J}+T_{\#}$, and then (2.9) is equivalent to

$$
\left\{\begin{array}{l}
\frac{d v}{d t}+G(v) v=\oint T_{J} f, v(0)=v_{0} \\
\frac{\partial T_{J}}{\partial t}+v \frac{\partial T_{J}}{\partial x}=h_{J}(x), T_{J}(0)=T_{0 J} \\
\frac{\partial T_{\#}}{\partial t}+v \frac{\partial T_{\#}}{\partial x}=h_{\#}(x), T_{\#}(0)=T_{0 \#}
\end{array}\right.
$$


and the equation for $T_{\#}$ is a linear nonautonomous PDE, once the subsystem of the first two equations is solved. Therefore, we have Proposition 3.3.

Proposition 3.10. If the set $J$ is finite, then the first two equations in (3.13) are equivalent to the finite-dimensional system

$$
\left\{\begin{array}{l}
\frac{d v}{d t}+G(v) v=\sum_{k \in J} a_{k}(t) \bar{c}_{k}, \\
\dot{a}_{k}(t)+2 \pi i k v(t) a_{k}(t)=b_{k}, \quad k \in J
\end{array}\right.
$$

where $a_{-k}=\bar{a}_{k}, b_{-k}=\bar{b}_{k}$, and $c_{-k}=\bar{c}_{k}$ which is equivalent to a system of ODEs in $\mathbb{R}^{N}$ with $N=|J|+1$ an odd number.

The remaining modes of the solution of (2.9), i.e., the modes of the component $T_{\#}$, are determined by the solution of

$$
\dot{a}_{k}(t)+2 \pi i k v(t) a_{k}(t)=b_{k}, \quad k \notin J
$$

and initial data $a_{k}(0) \in \mathbb{C}$.

On the other hand, assume also that the heat flux is given by

$$
h(x)=\sum_{k \in K} b_{k} e^{2 \pi k i x}, \quad K \subset \mathbb{Z}^{*} .
$$

Then we have the following result that, in particular, applies to the case of insulated boundaries, i.e., $h(x)=0$.

Proposition 3.11. Assume $K \cap J=\emptyset$; then the first two equations in (3.13) are equivalent to a second-order nonlinear damped ODE of the form

$$
e^{\prime \prime}(t)+G\left(e^{\prime}(t)\right) e^{\prime}(t)+F(e(t))=0
$$

with $F(e)=-\oint T_{0 J}(x-e) f(x) d x$ and the $\omega$-limit set of any solution of the ODE is included in the set of equilibria $\{(e, 0), F(e)=0\}$.

In particular, the solution of (3.13) verifies $v(t) \rightarrow 0$, and for every sequence $t_{n} \rightarrow \infty$ there exists a subsequence that we still denote $t_{n}$ such that

$$
T_{J}\left(t_{n}, x\right) \rightarrow T_{0 J}(x-e),
$$

where $e$ is such that $\oint T_{0 J}(x-e) f(x) d x=0$. If the zeros of $F$ are isolated then $e$ is independent of the sequence we choose and then

$$
T_{J}(t, x) \rightarrow T_{0 J}(x-e)
$$

as $t \rightarrow \infty$.

Proof. Since $K \cap J=\emptyset, h_{J}=0$ in (3.13) and we have $T_{J}(t, x)=T_{0 J}\left(x-\int_{0}^{t} v\right)$ and the equation for the velocity reads

$$
\frac{d v}{d t}+G(v) v=\oint T_{0 J}\left(x-\int_{0}^{t} v\right) f(x) d x .
$$

Now define $e(t)=\int_{0}^{t} v$; then $e(0)=0$ and $e^{\prime}(0)=v_{0}$ and

$$
e^{\prime \prime}(t)+G\left(e^{\prime}(t)\right) e^{\prime}(t)+F(e(t))=0,
$$


where the force term is $F(e)=-\oint T_{0 J}(x-e) f(x) d x$ and the potential is $V(e)=$ $\int_{0}^{e} F(r) d r$. Note that $F$ is continuous, 1-periodic and $\int_{0}^{1} F(r) d r=-\oint f(x) \int_{0}^{1} T_{0 J}(x-$ $r) d r d x=(\oint f)\left(\oint T_{0 J}\right)=0$. Therefore, $V$ is a bounded and periodic potential. Finally, observe that $F(e)=0$ for all $e \in \mathbb{R}$ if and only if $T_{0 J}=0$ and in this case $T_{J}(t, x)=0$ and $v(t) \rightarrow 0$ exponentially. Therefore, we assume henceforth that $F(e)$ is not identically zero.

The energy $E\left(e, e^{\prime}\right)=\frac{\left|e^{\prime}\right|^{2}}{2}+V(e)$ is a Lyapunov function for the nonlinear secondorder differential equation above, since

$$
\dot{E}(t)+G\left(e^{\prime}(t)\right)\left(e^{\prime}(t)\right)^{2}=0
$$

and $\dot{E}=0$ only for equilibrium points, i.e., for $e$ such that $F(e)=0$ and $e^{\prime}=$ 0 . Also, since $V$ is bounded below, the $\lim _{t \rightarrow \infty} E(t)$ always exists and moreover $\int_{0}^{\infty} G\left(e^{\prime}(t)\right)\left(e^{\prime}(t)\right)^{2} d t<\infty$.

From La Salle's invariance principle, if a solution is bounded, then its $\omega$-limit set is contained in the set of equilibria. Therefore, we must show that there are no unbounded solutions. Note that since the energy is bounded above and below, $e^{\prime}(t)$ is always bounded.

Let $V_{0}$ be the maximum value of the potential $V(e)$ and consider the compact set $I=\left\{\left(e, e^{\prime}\right), E\left(e, e^{\prime}\right) \leq V_{0}\right\}$. Then $I$ is invariant for the ODE, contains the real axis, is invariant under translations $e \mapsto e+1$, and contains the set of equilibrium points $\{(e, 0), F(e)=0\}$. Moreover the subset of equilibria $\left\{(e, 0), V(e)=V_{0}\right\}$ separates the connected components of the interior of $I$.

If a solution that is not an equilibrium enters $I$ in finite time, then it enters the interior of $I$ and remains in one of the connected components. Consequently the solution remains bounded and La Salle's principle applies. Assume there is a solution such that $\lim _{t \rightarrow \infty} E(t) \geq V_{0}+\epsilon$ for some $\epsilon>0$. Then $\left|e^{\prime}(t)\right|^{2} \geq 2 \epsilon$ and this is absurd, since $G_{0} \int_{0}^{\infty}\left(e^{\prime}(t)\right)^{2} d t \leq \int_{0}^{\infty} G\left(e^{\prime}(t)\right)\left(e^{\prime}(t)\right)^{2} d t<\infty$.

Therefore, assume there exists an unbounded solution such that $\lim _{t \rightarrow \infty} E(t)=$ $V_{0}$. We fix $e_{0}>0$; then there exists a sequence $t_{n} \rightarrow \infty$ such that $\left|e\left(t_{n}\right)\right|=e_{0}+n=e_{n}$. From the energy we obtain

$$
E\left(t_{n+1}\right)-E\left(t_{n}\right)=\frac{\left|e^{\prime}\left(t_{n+1}\right)\right|^{2}}{2}-\frac{\left|e^{\prime}\left(t_{n}\right)\right|^{2}}{2}=-\int_{t_{n}}^{t_{n+1}} G\left(e^{\prime}(s)\right)\left(e^{\prime}(s)\right)^{2} d s \rightarrow 0
$$

as $n \rightarrow \infty$. But on the other hand $\left|e^{\prime}(t)\right|^{2} \geq 2\left(V_{0}-V(e(t))\right)$ and integrating on $\left(t_{n}, t_{n+1}\right)$ we obtain

$$
\int_{0}^{1} \sqrt{2\left(V_{0}-V(z)\right)} d z=\int_{e_{n}}^{e_{n+1}} \sqrt{2\left(V_{0}-V(z)\right)} d z \leq \int_{t_{n}}^{t_{n+1}}\left(e^{\prime}(s)\right)^{2} d s \rightarrow 0,
$$

where we have used the change of variables $z=e(s)$ in the integral to the right and the fact that $V$ is periodic of period 1. Therefore, we have a contradiction and then all solutions enter the set $I$ and La Salle's principle applies.

The rest follows from the definition of the $\omega$-limit set and the fact that it is included in the set of equilibria. If $t_{n} \rightarrow \infty$, for some subsequence that we still denote by $t_{n},\left(e\left(t_{n}\right), e^{\prime}\left(t_{n}\right)\right) \rightarrow(e, 0)$, so $F(e)=0$, but this implies $v\left(t_{n}\right) \rightarrow 0$ and $T\left(t_{n}, x\right) \rightarrow$ $T_{0 J}(x-e)$. Since the limit for the velocity is independent of the subsequence, we have $v(t) \rightarrow 0$ and the rest is obvious.

Remark 3.1. Observe that if $K \cap J=\emptyset$, then Proposition 2.1 applies and this shows that the component $T_{\#}$ becomes unbounded. 
Also, note that in the proposition above the second-order ODE depends on the projection of the initial data $T_{0 J}$.

Remark 3.2. If $h_{J}(x)$ is nonzero, then $T_{J}(t, x)=T_{0 J}\left(x-\int_{0}^{t} v\right)+\int_{0}^{t} h_{J}\left(x-\int_{s}^{t} v\right) d s$ and the equation for the velocity is now

$$
\frac{d v}{d t}+G(v) v=\oint T_{0 J}\left(x-\int_{0}^{t} v\right) f(x) d x+\oint \int_{0}^{t} h_{J}\left(x-\int_{s}^{t} v\right) f(x) d s d x .
$$

Now we have

$$
\begin{aligned}
\oint \int_{0}^{t} h_{J}\left(x-\int_{s}^{t} v\right) f(x) d s d x & =\int_{0}^{t} \oint h_{J}\left(x-\int_{s}^{t} v\right) f(x) d x d s \\
& =\int_{0}^{t} \oint h_{J}\left(x+\int_{0}^{s} v\right) f\left(x+\int_{0}^{t} v\right) d x d s
\end{aligned}
$$

and then using again $e(t)=\int_{0}^{t} v$ we have

$$
e^{\prime \prime}(t)+G\left(e^{\prime}(t)\right) e^{\prime}(t)+F(e(t))=H(t, e(t))
$$

with $H(t, e(t))=\int_{0}^{t} J(e(t), e(s)) d s$ and $J(y, z)=\oint f(x+y) h_{J}(x+z) d x$, which is a damped nonlinear second-order differential equation "with memory."

4. Numerical results. In this section we describe the results of numerical experiments that were obtained using the five mode model. These experiments illustrate some of the interesting behavior in the model but do not represent an exhaustive numerical exploration. In particular, these experiments show that Lorenz-like behavior is not restricted to the case where $G$ is constant. All simulations were performed on a Silicon Graphics Indigo ${ }^{2}$ workstation in double precision with machine epsilon $\epsilon_{M} \approx 2.2 \times 10^{-16}$. The simulations were performed using a fourth-order implicit Runge-Kutta method, the fourth-order Gauss-Legendre method. Variable timestepping was employed by comparing the approximation obtained using two half steps with the approximation obtained using one full step to satisfy a local error tolerance of $10^{-6}$. The nonlinear equations obtained after discretization were solved using a full Newton iteration with LAPACK subroutines used to solve the resulting linear systems.

We assume that $K \cap J=\{ \pm k, \pm j\}$, and changing variables as $a_{n} \rightarrow a_{n} \bar{c}_{n}$ and $b_{n} \rightarrow b_{n} \bar{c}_{n}$ for $n=k, j$ and using real and imaginary parts, we obtain from (1.2)

$$
\begin{aligned}
v^{\prime}+G(v) v-2 x-2 u & =0, \\
x^{\prime}+\nu 4 \pi^{2} k^{2} x-2 \pi k v y & =A, \\
y^{\prime}+2 \pi k v x+\nu 4 \pi^{2} k^{2} y & =B, \\
u^{\prime}+\nu 4 \pi^{2} j^{2} u-2 \pi j v w & =C, \\
w^{\prime}+2 \pi j v u+\nu 4 \pi^{2} j^{2} w & =D,
\end{aligned}
$$

where $a_{k}(t)=x(t)+i y(t), b_{k}=A+i B$, and $a_{j}(t)=u(t)+i w(t), b_{j}=C+i D$. Note that the three mode model is obtained by making $C=D=u=w=0$.

We consider $G(v)=|v|+10^{-4}$ and investigate the interaction between the first and second temperature modes. We use $\nu$ as a tuning parameter, take $k=1$ and $j=2$, and set $A=C=0, B=30, D=20$. The initial conditions used are $v(0)=0$, $x(0)=-0.1, y(0)=1, u(0)=10^{-2}$, and $w(0)=1$.

Although the system is not dissipative when $\nu=0$ we observed numerically stable bounded orbits, Figure 1(a). As $\nu$ is increased we observed Lorenz-type behavior in 


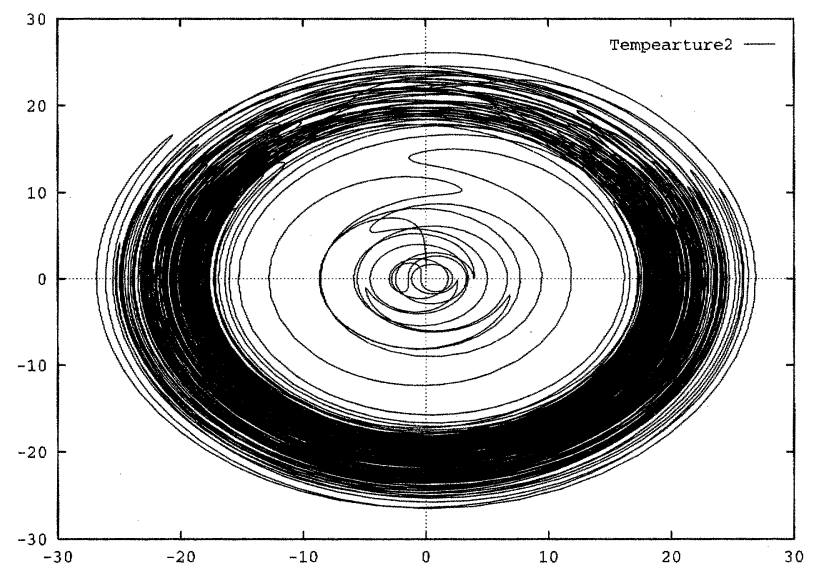

(a)

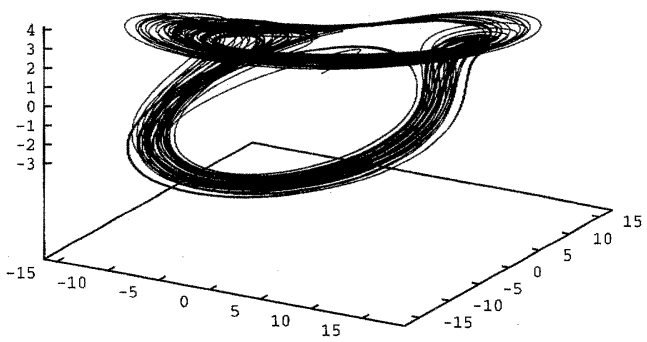

(b)

FIG. 1. (a) $(u, w)$ plot for $\nu=0.0$ and (b) $(x, y, v)$ plot for $\nu=1.0 \times 10^{-2}$.

Figure 1 (b) for $\nu=1.0 \times 10^{-2}$. At $\nu=1.5 \times 10^{-2}$ in Figure 2(a) convergence to a periodic solution was observed. Again we observed Lorenz-type behavior in Figure 2(b) for $\nu=2.5 \times 10^{-2}$. For large values of $\nu$ convergence to equilibria was observed.

5. Discussion. In this paper a closed loop thermosyphon model with and without diffusion and with a prescribed heat flux at the loop wall is analyzed. The existence of an attractor and an inertial manifold is proven, and an exact explicit reduction to a low-dimensional system is obtained. The reduced models are odd dimension ODEs and contain as a special case a three-dimensional model that corresponds exactly to the Lorenz equations in the case of linear friction. Also, the form of the reduced system allows for suitable design of geometries and/or heat fluxes to produce any desired odd number of variables. Moreover, the Fourier coefficients of the geometry 


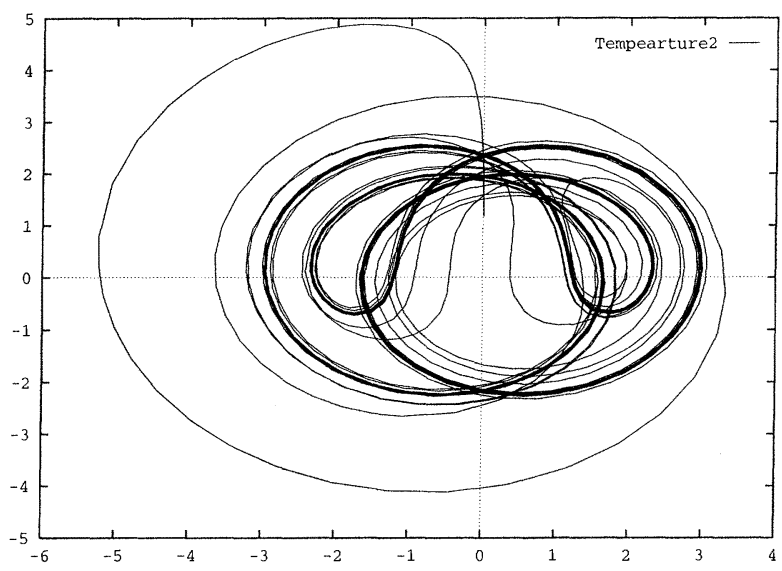

(a)

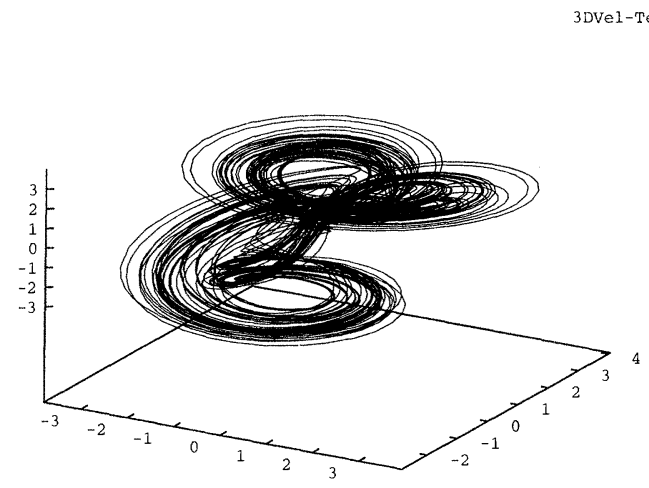

(b)

FIG. 2. (a) $(u, w)$ plot for $\nu=1.5 \times 10^{-2}$ and (b) $(u, w, v)$ plot for $\nu=2.5 \times 10^{-2}$.

and heat flux can be chosen such that, in combination with the diffusion coefficient, the reduced model contains interesting nontrivial complex dynamics.

There are several questions that require further analysis. For example, an estimate of the Hausdorff or fractal dimension of the attractor in terms of physically relevant quantities would be of interest. Also, the existence of a low-dimensional inertial manifold when the set $K \cap J$ is not finite would be of interest. In addition, more numerical exploration is needed on five and higher mode models to obtain, in particular, an understanding of the dependence of the dimension of the attractor on the number of modes and of bifurcations produced as the diffusion coefficient and other parameters are varied.

Acknowledgments. We are grateful to the referees for several helpful remarks. 


\section{REFERENCES}

[1] A. M. Bloch AND E. S. TITI, On the dynamics of rotating elastic beams, in New Trends in System Theory, Progr. Systems Control Theory 7, Birkhäuser Boston, Boston, MA, 1991, pp. 128-135.

[2] K. CHEN, On the oscillatory instability of closed-loop thermosyphons, J. Heat Transfer, 107 (1985), pp. 826-832.

[3] J. H. CuRRY, A generalized Lorenz system, Comm. Math. Phys., 60 (1978), pp. 193-204.

[4] C. FoIAs, G. SELL, AND R. TEMAM, Inertial manifolds for nonlinear evolutionary equations, J. Differential Equations, 73 (1988), pp. 309-353.

[5] R. GREIF, Y. ZVIRIN, AND A. MERTOL, The transient and stability behavior of a natural convection loop, J. Heat Transfer, 101 (1979), pp. 684-688.

[6] J. K. HALE, Asymptotic Behavior of Dissipative Systems, AMS, Providence, RI, 1988.

[7] J. E. HART, Observations of complex oscillations in a closed thermosyphon, J. Heat Transfer, 107 (1985), pp. 833-839.

[8] D. HENRY, Geometric theory of semilinear parabolic equations, Lecture Notes in Math. 840, Springer-Verlag, Berlin, New York, 1982.

[9] M. A. Herrero and J. J. L. Velázquez, Stability analysis of a closed thermosyphon, European J. Appl. Math., 1 (1990), pp. 1-24.

[10] D. JAPIKSE, Advances in thermosyphon technology, Adv. Heat Transfer, 9 (1973), pp. 1-111.

[11] J. B. Keller, Periodic oscillations in a model of thermal convections, J. Fluid Mech., 26 (1966), pp. 599-606.

[12] A. LiÑÁN, Analytical description of chaotic oscillations in a toroidal thermosyphon, in Fluid Physics, Lecture Notes of Summer Schools, M. G. Velarde and C. I. Christov, eds., World Scientific, River Edge, NJ, 1994, pp. 507-523.

$[\because \rightarrow$ E. N. Lorenz, Deterministic nonperiodic flow, J. Atmospheric Sci., 20 (1963), pp. $130-141$.

[14] A. Rodríguez-Bernal, Attractors and inertial manifolds for the dynamics of a closed thermosyphon, J. Math. Anal. Appl., 193 (1995), pp. 942-965.

[15] A. Rodríguez-Bernal, Inertial manifold for dissipative semiflows in Banach spaces, Appl. Anal., 37 (1990), pp. 95-141.

[16] A. Rodríguez-Bernal ANd E. S. VAN VleCK, Complex oscillations in a closed thermosyphon, Internat. J. Bifur. Chaos Appl. Sci. Engrg., 8 (1998).

[17] C. Sparrow, The Lorenz Equations: Bifurcation, Chaos, and Strange Attractors, SpringerVerlag, Berlin, New York, 1982.

[18] A. M. Stuart, Perturbation theory for infinite-dimensional dynamical systems, in Theory and Numerics of Ordinary and Partial Differential Equations, M. Ainsworth, J. Levesley, W. A. Light, and M. Marletta, eds., Oxford University Press, Oxford, UK, 1994.

[19] J. J. L. VelázQUez, On the dynamics of a closed thermosyphon, SIAM J. Appl. Math., 54 (1994), pp. 1561-1593.

[20] P. Welander, On the oscillatory instability of a differentially heated fluid loop, J. Fluid Mech., 29 (1967), pp. 17-30. 